\title{
Prevalence and progression of aortic dilatation in adult patients with Turner syndrome: a cohort study
}

\author{
Bruno Donadille, ${ }^{1,2}$, Sophie Tuffet ${ }^{3}$, Clement Cholet ${ }^{4}$, Mariana Nedelcu ${ }^{1,2}$, Nathalie Bourcigaux ${ }^{1,2}$, \\ Laurence Iserin ${ }^{5}$, Laurence Monnier-Cholley ${ }^{4}$, Alexandra Rousseau ${ }^{3}$ and Sophie Christin-Maitre ${ }^{1,2,6}$
}

${ }^{1}$ Endocrinology Department, Saint Antoine Hospital and Sorbonne Université, Assistance Publique-Hôpitaux de Paris, Paris, France, ${ }^{2}$ Centre de Référence des Maladies Endocriniennes Rares de la Croissance et du Développement (CRMERCD), Filière FIRENDO, Endo-ERN id 739527, Paris, France, ${ }^{3}$ Clinical Research Unit (URC-EST), Saint Antoine Hospital and Functional Unit of Pharmacology, Sorbonne Université, Paris, France, ${ }^{4}$ Radiology Department, Saint Antoine Hospital, Assistance Publique-Hôpitaux de Paris, Sorbonne Université, Paris, France, ${ }^{5}$ Congenital Heart Disease Unit, Cardiology Department, Hôpital Européen Georges Pompidou, Assistance Publique-Hôpitaux de Paris, Paris, France, and ${ }^{6}$ Inserm Unit UMR-S933, Paris, France

Correspondence should be addressed to B Donadille Email bruno.donadille@aphp.fr

\section{Abstract}

Objective: Turner syndrome (TS) is a rare disorder affecting 1/2500 female newborn. Aortic dilatation (AD) and aortic dissection represent a major concern in TS. The aims of our study were to describe the aortic root growth, potential aortic dilatation (AD) risk factors and cardiovascular outcomes in a cohort of patients with TS.

Methods: Among 204 adult patients included, 197 were studied using a standardized 1.5 Tesla MRI protocol. AD was defined as an aortic diameter $\geq 20 \mathrm{~mm} / \mathrm{m}^{2}$ at the Valsalva sinuses and/or at the ascending aorta, when indexed to body surface area.

Results: At baseline, AD was present in 81/197 (41.1\%) and 32/197 (16.2\%) of patients, at the levels of Valsalva and ascending aorta, respectively. The aortic Valsalva diameter was larger in patients treated for thyroiditis $(P<0.001)$. Potential risk factors of AD were aging $(P<0.001)$ and the presence of bicuspid aortic valve (BAV) $(P=0.002)$. The hazard ratio (HR) of AD occurrence in the presence of BAV was $2.2(95 \% \mathrm{Cl}$ : 1.33-3.71). After a median follow-up period of 5.1 years $(n=143)$, AD was present in 58/143 (40.6\%) and 25/143 (17.5\%) of patients at the levels of Valsalva and ascending aorta, respectively. The median aortic growth of the Valsalva sinuses remained stable. At the ascending aorta, it increased by $0.14 \pm 0.61 \mathrm{~mm} /$ year. Only one aortic-related death was observed.

Conclusion: $\mathrm{AD}$ is common in adult patients with TS. However, our results are rather reassuring, as the median aortic diameters remained stable after 5.1 years and few aortic events were observed.

\section{Introduction}

Turner syndrome (TS) is a rare disorder (ORPHA \#881) which affects $1 / 2500$ female newborns and is characterized by the complete or partial loss of the second $\mathrm{X}$ chromosome. The associated clinical conditions include growth failure $(95-100 \%$ of cases), primary ovarian insufficiency (95\%), autoimmune disease (58\%) as well as congenital or acquired cardiovascular disease (1).
Congenital cardiovascular malformations are present in about half of patients with TS (2). The most frequent one is a bicuspid aortic valve (BAV), occurring in $20-30 \%$ of TS (3). In the general population, its prevalence is $2-3 \%$ in males and $0.05 \%$ in females (4). It has been reported that the prevalence of BAV and other congenital cardiovascular malformations are more

Published by Bioscientifica Ltd. 
frequent in patients with a homogenous 45,X karyotype than in patients with $45, \mathrm{X} / 46, \mathrm{XX}$ mosaicism $(1,5)$. BAV is associated with proximal aortic dilatation (AD) and is a predisposing risk factor for subsequent aortic dissection (2). In patients with BAV or Marfan syndrome, betablocker or losartan treatments are recommended when $\mathrm{AD}$ is detected $(2,6)$.

The mortality of patients with TS is increased three-fold when compared to the general population $(\mathrm{SMR}=3.0$; 95\% CI 2.7-3.4) (7), in association with the occurrence of cardiovascular diseases including aortic dissection. The incidence of aortic dissection diagnosed by echocardiography in a cohort of Swedish women with TS, after 23 years of follow-up, was 110 cases/100 000 patient years (8). The risk factors of aortic dissection, in this study, were the presence of $\mathrm{AD}$, hypertension, $\mathrm{BAV}$ and aortic coarctation. However, in $11 \%$ of the cases reported in the literature, TS by itself was an independent risk factor of aortic dissection (9). European guidelines concerning screening for AD in TS have been published in 2017 (2). They recommend measuring aortic root diameters at reference levels, such as the aortic annulus, Valsalva sinuses, sinotubular junction as well as ascending aorta and indexing the aortic diameter to the adult patient's body surface area (BSA) (2). The primary objective of our study was to describe the aortic root growth using standardized screening by MRI in a large cohort of adult patients with TS. The secondary objectives were to identify risk factors of $\mathrm{AD}$ and to describe the cardiovascular outcomes.

\section{Patients and methods}

\section{Patients}

Adult patients with TS were recruited in our unit, labeled by the French Ministry of Health as a Reference Center for Rare diseases of Growth Disorders and development since 2006. This center belongs to the national endocrine rare disease network named FIRENDO (firendo.fr), and to the Endo-ERN network (endo-ern.eu; id 739527).

To be included, each patient had a standard karyotype on blood cells with at least $10 \%$ of cells displaying a total or partial loss of one $\mathrm{X}$ chromosome. This observational study was approved by the Paris Nord Ethics Review Committee for Biomedical Research Projects (CEERB) (No 12-029). All the patients' files were included in the CEMARA database. This national database has been declared to the French data protection agency (Commission Nationale de l'Informatique et des Libertés (CNIL), No 909474 in 2010). In compliance with French law, consent of non-opposition to collect and use the data was obtained from each patient.

Data collected for the present study included the patient's date of birth, auxological data, karyotype and previous history of surgery. Congenital heart malformations, such as a bicuspid aortic valve (BAV) or other valvulopathies and aortic coarctation, were recorded as well as past and present medical treatments. BSA was calculated according to Dubois' formula (10).

Automated office blood pressure readings were obtained in the seated position, with an appropriate cuff size at the level of the patient's right atrium, as recommended (2). Diagnosis of thyroiditis was established by TSH level and/or the presence of anti-thyroid antibodies (antithyroperoxydase and anti-thyroglobulin), as recommended (2). When patients were treated with L-thyroxin at the time of inclusion, they were defined as under treatment.

\section{Imaging}

All patients were scanned using the same acquisition protocol for baseline and follow-up imaging with a 1.5 Tesla MRI (Signa HDxt; GE Medical Systems). A standard non-contrast MRI protocol with a dedicated phased-array cardiac coil and retrospective ECG gating was performed. Gradient echo localizers were used to obtain anatomic axes of the aorta. Cine b-SSFP imaging was performed in coronal oblique view (or left ventricular outflow tract 2 LVOT 2 view), sagittal oblique view and axial oblique view centered on the aortic valve. Aortic diameters were assessed for all aortic MRI scans by experienced cardiovascular radiologists on a dedicated workstation (Carestream Health, Rochester, NY) using the coronal oblique and sagittal oblique cine images at the Valsalva sinuses and tubular ascending aorta. Measures were obtained using the classic inner edge to inner edge method in diastole (11). Aortic valve morphology and assessment for aortic regurgitation and/or stenosis were evaluated with cine axial oblique images. The intraclass correlation coefficient (ICC) and its 95\% CIs (estimated by bootstrap) were used to assess the concordance of measurements between three operators from the same radiology department for Valsalva and ascending aorta measurements in a sample of patients: the ICC are 0.83 (95\% CI 0.35-0.90) and 0.84 (95\% CI 0.47-0.92) for MRI measurements at the Valsalva level and at the ascending aorta level, respectively.

\section{Aortic dilatation}

$\mathrm{AD}$ was defined as an absolute aortic diameter at the Valsalva sinuses and/or at the ascending aorta larger than 
$32 \mathrm{~mm}$ and/or $\geq 20 \mathrm{~mm} / \mathrm{m}^{2}$, when indexed to BSA. Aortic systolic index (ASI) was defined as the ascending aorta diameter indexed to BSA (2).

\section{Aortic root growth}

When at least two MRIs were available, kinetics of aortic root diameters were systematically assessed at the Valsalva sinuses and ascending aorta. The rate of progression was expressed in millimeter per year (mm/year) and was defined as: (last MRI aortic diameter - first MRI aortic diameter $(\mathrm{mm})) /($ elapsed time between the last and the first MRI (year)). As previously described, the threshold of $1 \mathrm{~mm}$ /year was used to define rapid aortic growth (12).

\section{Statistical analysis}

Baseline characteristics were expressed as frequency and percentage for categorical variables and as median (interquartile range, IQR) for continuous variables. Mean baseline absolute aortic diameter at the Valsalva level was compared between patients according to the presence of BAV and/or treated thyroiditis using a oneway ANOVA test. Mean baseline aortic diameter was also compared according to treated thyroiditis in patients with and without BAV by using Student's t-test (Bonferroni correction, $P$-value $<0.025)$.

AD-free survival, according to the presence of BAV, was represented using Kaplan-Meier survival curves in the population with at least one informative MRI. Risk factors of $\mathrm{AD}$ occurrence were evaluated using a Cox proportional hazard model. Age, interaction variable between age at diagnosis of TS and past GH treatment were forced in the model. Presence of BAV, past aortic surgery, aortic coarctation, a homogenous 45,X karyotype and treated cardiovascular risk factors were selected in univariate analysis ( $P$-value $<0.20)$. Proportional hazard and log-linearity hypothesis have been checked. Results were expressed as hazard ratios for Cox models with 95\% CIs.

Progression of the absolute aortic diameter at the Valsalva sinuses and the ascending aorta was studied in the population with at least two informative MRIs using a linear regression model. Final models were built using a backward stepwise procedure with covariates selected in the univariate analysis ( $P$-value $<0.20)$. Normality of the aortic diameter progression, log-linearity hypothesis, homoscedasticity and independence of residues have been checked. All analyses were performed with the SAS version 9.4 statistical software (SAS Institute Inc., Cary, NC, USA).

\section{Results}

\section{Baseline characteristics}

Two hundred and four women were included in the cohort between 2006 and 2019. Among them, a standardized aortic MRI scan protocol at the two aortic positions studied was at least performed once for 197 patients and twice for 143 patients (Fig. 1). Baseline clinical characteristics, karyotypes, medical histories, past and current treatments among 197 patients are reported in Table 1. Other observed valvulopathies were aortic insufficiencies (4.9\%) and aortic stenosis (0.5\%). Cardiovascular surgery had been performed during childhood in 12/197 (6.1\%), including

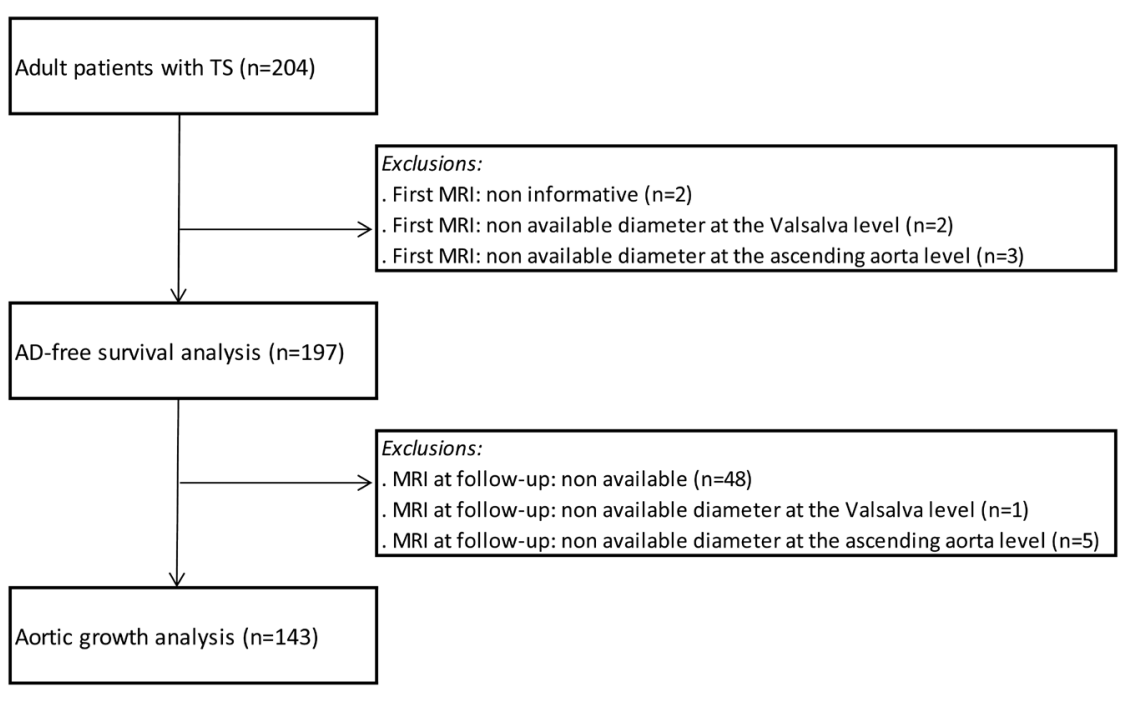

Figure 1

Flow chart of patients included in the study. 
Table 1 Baseline clinical characteristics of the cohort ( $n=197$ patients with TS) in the aortic dilatation (AD)-free survival analysis. Results are expressed as $n(\%)$, or median (IQR).

\begin{tabular}{|c|c|}
\hline & $\begin{array}{l}\text { AD-free survival analysis } \\
\text { population }(n=197)\end{array}$ \\
\hline Age (years) & $25.6(19.6 ; 34.2)$ \\
\hline Years since diagnosis & $18.0(10.0 ; 24.0)$ \\
\hline \multicolumn{2}{|l|}{ Karyotype } \\
\hline $45, x$ & $79(40.1)$ \\
\hline $45, X / i(X q)+$ & $32(16.2)$ \\
\hline $45, X / 46, X X$ & $21(10.7)$ \\
\hline $45, X / 46, X r(X)$ & $20(10.2)$ \\
\hline $45, X / Y+$ & $10(5.1)$ \\
\hline $45, X / 47, X X X$ & $7(3.6)$ \\
\hline Others & $28(14.2)$ \\
\hline \multicolumn{2}{|l|}{ Anthropometric values } \\
\hline Height $(\mathrm{cm})$ & $152.0(147.0 ; 157.0)$ \\
\hline Weight (kg) & $52.0(46.0 ; 60.0)$ \\
\hline BMI $\left(\mathrm{kg} / \mathrm{m}^{2}\right)$ & $22.8(20.3 ; 25.7)$ \\
\hline $\mathrm{BSA}\left(\mathrm{m}^{2}\right)$ & $1.5(1.4 ; 1.6)$ \\
\hline $\begin{array}{l}\text { Median blood pressure level } \\
(\mathrm{mmHg})\end{array}$ & $110 / 70(102 / 63 ; 120 / 77)$ \\
\hline \multicolumn{2}{|l|}{ Medical history } \\
\hline Tabagism & $23(11.9)^{c}$ \\
\hline Diabetes (antidiabetic drug) & $12(6.1)^{a}$ \\
\hline Dyslipidemia (lipid-lowering drug) & $10(5.1)^{\mathrm{a}}$ \\
\hline $\begin{array}{l}\text { Coronaropathy } \\
\text { (medical treatment) }\end{array}$ & $3(1.5)$ \\
\hline $\begin{array}{l}\text { Hypertension } \\
\text { (antihypertensive drug) }\end{array}$ & $9(4.6)$ \\
\hline Past treatment with $\mathrm{GH}$ & $147(75.0)^{\mathrm{a}}$ \\
\hline Thyroidal supplementation & $65(33.2)^{a}$ \\
\hline $\begin{array}{l}\text { Sexual hormone replacement } \\
\text { therapy }\end{array}$ & $177(90.8)^{b}$ \\
\hline Aortic coarctation & $16(8.1)$ \\
\hline Bicuspid aortic valve (BAV) & $45(22.8)$ \\
\hline Valvulopathies different from BAV & $24(12.2)$ \\
\hline \multicolumn{2}{|l|}{ Basal aortic diameters } \\
\hline \multicolumn{2}{|l|}{ Valsalva level } \\
\hline$\left(\mathrm{mm} / \mathrm{m}^{2}\right)$ & $18.9(17.2 ; 20.9)$ \\
\hline Dilatation & $81(41.1)$ \\
\hline \multicolumn{2}{|l|}{ Ascending aorta level } \\
\hline$\left(\mathrm{mm} / \mathrm{m}^{2}\right)$ & $15.9(14.5 ; 17.7)$ \\
\hline Dilatation & $32(16.2)$ \\
\hline
\end{tabular}

Results are expressed as $n(\%)$, or median (IQR). Missing values ${ }^{a} n=1$; ${ }^{\mathrm{b}} n=2 ;{ }^{\mathrm{c}} n=3$.

isolated aortic coarctation repairs ( $n=6$ patients). Sixtyfive patients had thyroiditis. Among them, 43 were positive for anti-thyroid antibodies.

Median baseline aortic diameter at the Valsalva level was $18.9 \mathrm{~mm} / \mathrm{m}^{2}$ (IQR: 17.2-20.9) (Fig. 2A). Median ASI was $15.9 \mathrm{~mm} / \mathrm{m}^{2}$ (IQR: 14.5-17.8) (Fig. 2B). At baseline, $83 / 197$ (42.1\%) patients had $\mathrm{AD}$ : it was observed in 81/197 (41.1\%) and 32/197 (16.2\%) patients at the Valsalva and at the ascending aorta level, respectively. $\mathrm{AD}$ was observed at the ascending aorta but not at the Valsalva level in two cases. The median age at patients with baseline AD was 29.4 years (IQR: 20.0-37.4) and 34.8 years (IQR: 25.9-43.0) at the Valsalva and the ascending aorta levels, respectively. Baseline aortic diameter at the Valsalva level was significantly different according to the presence of bicuspid and/or treatment for thyroiditis $(P<0.001)$ (Fig. 3). In patients with BAV, aortic diameters at the level of Valsalva were larger in patients treated for thyroiditis $(31.8 \pm 4.5$ vs $30.8 \pm 3.7 ; P<0.001)$. In patients without BAV, a treated thyroiditis also led to larger aortic diameters $(28.5 \pm 3.7$ vs $27.0 \pm 3.1 ; P<0.001)$.

\section{Follow-up}

Aortic dilatation (AD)-free survival

AD was observed in $102 / 197$ (51.8\%) patients. The median survival time without $\mathrm{AD}$ was 35.0 years. $\mathrm{AD}$ occurrence was present in 100/197 (50.8\%) and 42/197 (21.3\%) patients at the Valsalva and the ascending aorta level, respectively. Using multivariate Cox analysis, both aging $(P<0.001)$ and the presence of BAV $(P=0.002)$ were significantly associated with AD occurrence. It was not associated with past GH treatment, the presence of aortic coarctation, or a $45, \mathrm{X}$ karyotype. Adjusted hazard ratio (HR) of AD occurrence in patients with BAV was $2.2(95 \%$ CI: 1.33-3.71), as compared to those without BAV (Fig. 4).

\section{Aortic growth}

In the cohort, 143 patients had at least 2 MRIs (Supplementary Table 1, see section on supplementary materials given at the end of this article). These patients were not different from the patients with one MRI (data not shown). Their median number of MRIs was 3.0 (IQR: 2.0-4.0) and their median follow-up was 5.1 years (IQR: 2.8-7.5). At the end of the follow-up period, $\mathrm{AD}$ was found in 58/143 (40.6\%) and 25/143 (17.5\%) at the level of the Valsalva sinuses and ascending aorta, respectively.

At the end of follow-up, mean progression of aortic diameter at the Valsalva sinuses was $0.0 \pm 0.80 \mathrm{~mm} /$ year. Results from a multivariate linear regression analysis showed an association between baseline aortic diameter, BSA and treated hypertension and aortic growth at the Valsalva $(P<0.05)$. At the end of the follow-up, mean progression of aortic diameter at the ascending aorta was $0.14 \pm 0.61 \mathrm{~mm} /$ year. According to a multivariate linear regression analysis, age, baseline aortic diameter and the presence of a valvulopathy different from uncomplicated BAV, were significantly associated $(P<0.05)$ with aortic growth. 

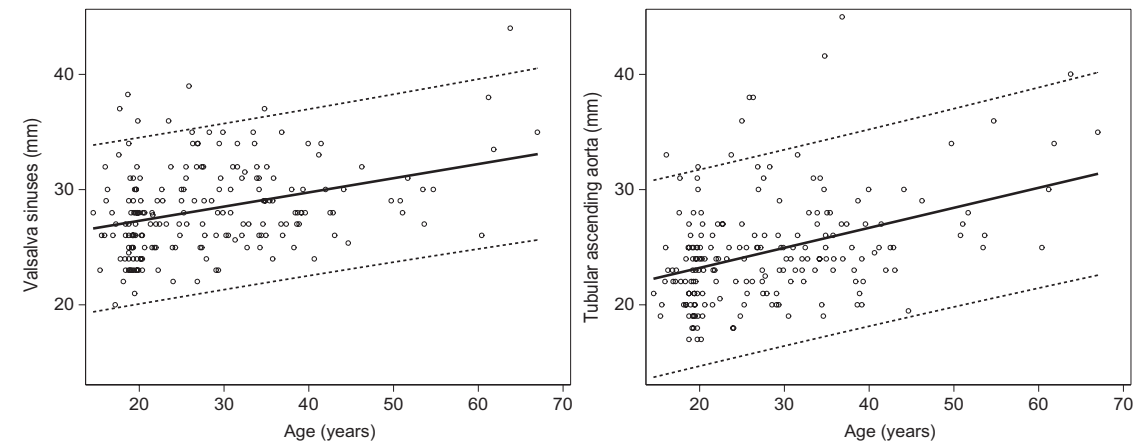

\section{Figure 2}

Scatterplot showing the baseline values of the aortic diameter $(\mathrm{mm})$ related to the patient's age (X-axis). Each dot represents a patient: $(A)$ at the Valsalva sinuses ( $n=197) ;(B)$ at the tubular ascending aorta $(n=197)$. The central line represents the regression line and the dotted lines represent the $95 \% \mathrm{Cl}$.

At the end of the study, four patients had a progression of their aortic diameter higher than $1 \mathrm{~mm} /$ year at the Valsalva sinuses and seven others at the level of ascending aorta. Ten of them had an aortic diameter above $25 \mathrm{~mm} / \mathrm{m}^{2}$ at a median age of 52 years (IQR: 36.5-65.7).

Of these 143 patients, 15 (10.5\%) were treated with beta-blockers. Among them, this treatment was prescribed in the presence of $\mathrm{AD}$ for eight patients. Their median age was 36.5 years (IQR: 29.7-40.0). The aortic diameter growth was not significantly different between patients with beta-blockers and those without beta-blocker intake, neither at the Valsalva sinuses $(P=0.18)$ nor at the ascending aorta $(P=0.33)$ levels.

During the follow-up period, 11 patients became pregnant, leading to 13 births. Aortic diameters before and after pregnancy were available for five patients (median age: 31.0 years old (IQR: $30-35)$ ). Their aortic growth (mm/ year) was not significantly different from the rest of the cohort, both at the Valsalva $(P=0.39)$ and the ascending aorta $(P=0.22)$ levels. The rate of rapid progression $(>1$ $\mathrm{mm}$ /year) was not different between pregnant and nonpregnant patients $(P=0.20)$.

\section{Cardiovascular outcomes}

During the follow-up period, two patients had prophylactic aortic surgery for AD. ASI values at the time of surgery were $38 \mathrm{~mm} / \mathrm{m}^{2}$ and $25 \mathrm{~mm} / \mathrm{m}^{2}$ for each of these patients.

Three patients had died at the end of the follow-up period. One death was related to an aortic dissection in a 65-year-old patient with a major $\mathrm{AD}(44 \mathrm{~mm}$ at the Valsalva sinuses; $30.13 \mathrm{~mm} / \mathrm{m}^{2}$ ). She had previously declined aortic surgery. The second death occurred in a 39-year-old patient who had cardiorespiratory arrest after surgery for a grade IV mitral insufficiency. The third death occurred after a second episode of stroke in a 70-yearold hypertensive patient. Finally, a 33-year-old patient presented a spontaneous dissection of her right vertebral artery. Her aortic diameter, measured 6 months before this vascular event, was $32 \mathrm{~mm}\left(19.9 \mathrm{~mm} / \mathrm{m}^{2}\right)$ at the Valsalva sinuses. She progressively recovered from this episode and completed the study.

\section{Discussion}

Our study evaluated the cardiovascular outcomes of a large cohort of adult patients with TS who had at least one 1.5 Tesla MRI, measuring aortic diameters both at the Valsalva and the ascending aorta levels. At baseline, AD was observed in 81/197 (41.1\%) and 32/197 (16.2\%) patients, at the Valsalva and ascending aorta levels, respectively. At the end of the follow-up period, AD was present in 58/143 (40.6\%) and 25/143 (17.5\%) at those same levels, respectively.

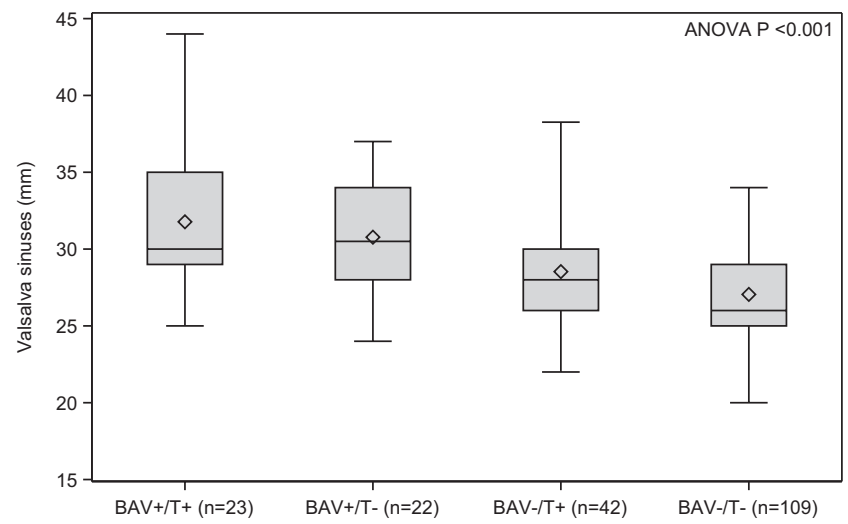

Figure 3

Baseline boxplot of aortic diameter at the Valsalva sinuses, according to the presence (+) or absence (-) of a bicuspid aortic valve (BAV) or a history of treated thyroiditis (T) $(n=197)$. The length of the box represents the interquartile range (distance between 25th and 75th percentiles); the symbol inside the box represents the mean; the horizontal line inside the box represents the median; and the vertical lines issuing from the box extend to the minimum and maximum values. 


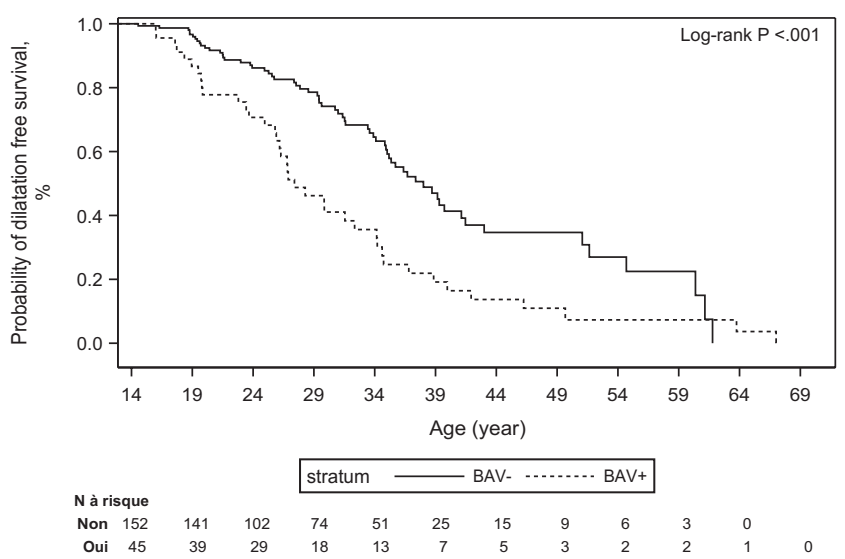

\section{Figure 4}

Kaplan-Meier survival estimator without aortic dilatation (AD) of the Valsalva sinuses according to age (X-axis), in the presence (black line) or the absence (dotted line) of bicuspid aortic valve (BAV) $(n=197)$.

We show that the presence of BAV was significantly associated with the occurrence of AD. Furthermore, we were able to quantify the risk of $\mathrm{AD}$ according to the presence of $\mathrm{BAV}$, with a hazard ratio (HR) of 2.2 (95\% CI 1.33-3.71). AD was found to be much more frequent at the Valsalva level than at the ascending aorta. In a cohort of 253 patients with TS, Sachdev et al. showed that 'typical' BAV, resulting in anterior and posterior cusps, was frequent and was correlated with $\mathrm{AD}$ at the Valsalva level (3). Therefore, a potential explanation of the highest rate of $\mathrm{AD}$ at the Valsalva level might rely on this type of BAV. Unfortunately, in our study, data concerning the type of BAV were not available for all patients.

In our study, the presence of a treated thyroiditis was associated with baseline AD. Regardless of the presence of BAV, the association remained significant $(P<0.001)$. Previous studies addressing congenital aortopathies have shown that systemic auto-immunity and inflammation are present in aortic remodeling. Indeed, proliferation of smooth muscle cells of the aortic media is regulated by cytokines secreted by immune and endothelial cells, whereas muscle remodeling involves the action of metalloproteases and TGF- $\beta$-mediated signaling (13). To our knowledge, the present study is the first one reporting a relationship between thyroiditis and $\mathrm{AD}$ in patients with TS.

Few studies have evaluated the progression of aortic diameters in adult patients with TS. Mortensen et al. (14) studied 91 patients for an average follow-up period of 8.8 years. The aortic progression was $0.32 \pm 0.3$ and $0.20 \pm 0.2 \mathrm{~mm} /$ year at the Valsalva sinuses and the ascending aorta level, respectively. Duijnhouwer et al. (15) studied 171 adult patients with TS, screened either by MRI or CT-scan for AD at the tubular ascending aorta level. This study reported a median aortic progression of $0.20 \mathrm{~mm} /$ year (IQR: 0.0-0.4) (15). Our results based on the evaluation of aortic diameters of both the Valsalva sinuses and the ascending aorta show a median increase in size of $0.0 \pm 0.80$ and $0.14 \pm 0.61 \mathrm{~mm} / \mathrm{year}$, respectively. Although $\mathrm{AD}$ is more frequent at baseline at the Valsalva level, the increase in aortic diameter size at this level is slower than that at the ascending aorta level. In our study, the progression of aortic diameters is lower than that reported previously. A potential explanation relies on the fact that our population has few cardiovascular risk factors and that median BMI was in the normal range $\left(22.8 \mathrm{~kg} / \mathrm{m}^{2}\right)$. A past history of ischemic heart disease is present in only $1.5 \%$ of the cohort and our population is young, since the median age is 25.6 years.

The multivariate linear regression analysis showed that the Valsalva diameter increases with hypertension $(P<0.05)$, strongly suggesting that this clinical condition enhances the development of AD. Duijnhouwer et al., using a similar statistical approach, showed that GH treatment was a risk factor for aortic diameter growth (15). However, this result is not confirmed in our study, since past $\mathrm{GH}$ treatment was related neither to baseline $\mathrm{AD}$ nor to progression of aortic diameters. After multivariate analysis, we found that aortic growth at the ascending aorta was associated with other valvulopathies different from uncomplicated BAV, mainly aortic insufficiency and/or stenosis. Therefore, aortic valve diseases might be involved in aortic diameter growth.

At the end of the follow-up period, 10/143 (7.0\%) patients experienced a progression of their aortic diameter $>1 \mathrm{~mm} /$ year. This prevalence is higher than that $(4.6 \%)$ reported in the Dutch study (15). This may be due to the fact that we measured two aortic positions, giving 2.8 and $4.9 \%$ of $\mathrm{AD}$ at the Valsalva and the ascending aorta, respectively. However, in our cohort, the number of patients with a rapid increase in their aortic diameter was too low for identifying risk factors for a rapid progression.

To our knowledge, the impact of beta-blocker treatments in patients with TS has not been previously reported. In patients with Marfan syndrome, beta-blockers slow the rate of $\mathrm{AD}$ (16). Among the patients treated with beta-blockers in the present study, aortic diameter growth did not significantly differ from that in patients without a beta-blocker therapy. However, our study is not powerful 
enough for demonstrating the protective role of betablockers in the development of AD.

Finally, during our follow-up period, the number of cardiovascular events was low (1.96\%) and only one death was related to an aortic dissection. In the Dutch cohort study with a 6.8-year follow-up (15), two deaths related to aortic dissection were recorded, both at the age of 44 years. An American study, including 317 women with a very long follow-up (mean 11 years), reported that cardiovascular diseases were the main cause of death in TS (17). Therefore, our median follow-up period of 5.1 years in a rather young population probably underestimated the rate of cardiovascular events.

Our study has some weaknesses. It could be argued that among our initial cohort, only 143 patients had performed several MRIs. However, their clinical characteristics were not different from these in the other patients included in the study. Furthermore, patients had up to eight scans with a maximum follow-up of 11.6 years. During follow-up, some patients had a decrease in the absolute aortic diameter. This could be due to the measurement accuracy of MRI. Concerning pregnant women, although their aortic diameter growth was not significantly different from that in the rest of the cohort, their number was too low for reassuring pregnant patients concerning AD.

In the near future, 4D MRI imaging and measurement of stiffness/distensibility of the proximal aorta should be available (18). Further genetic studies are needed to decipher the molecular mechanisms involved in BAV and $\mathrm{AD}$ (19) and, therefore, to improve cardiovascular care in patients with TS.

\section{Conclusion}

Aortic MRI in a population of adult patients with TS demonstrated a high rate of $\mathrm{AD}$, especially at the Valsalva sinuses. The rate of progression of aortic diameters during the follow-up period of 5 years was low. Few deaths related to cardiovascular events were observed. Although our data are reassuring, a long term screening is required to identify the few patients with a pejorative aortic outcome.

Supplementary materials

This is linked to the online version of the paper at https://doi.org/10.1530/ EJE-20-0284.

\section{Declaration of interest}

The authors declare that there is no conflict of interest that could be perceived as prejudicing the impartiality of this study.

\section{Funding}

This research did not receive any specific grant from any funding agency in the public, commercial or not-for-profit sector.

\section{Acknowledgements}

All authors would like to thank the patients with Turner syndrome followed in our hospital and the nurses from our department.

\section{References}

1 Gravholt CH, Viuff MH, Brun S, Stochholm K \& Andersen NH. Turner syndrome: mechanisms and management. Nature Reviews: Endocrinology 201915 601-614. (https://doi.org/10.1038/s41574-0190224-4)

2 Gravholt CH, Andersen NH, Conway GS, Dekkers OM, Geffner ME, Klein KO, Lin AE, Mauras N, Quigley CA, Rubin K et al. Clinical practice guidelines for the care of girls and women with Turner syndrome: proceedings from the 2016 Cincinnati International Turner Syndrome Meeting. European Journal of Endocrinology 2017 177 G1-G70. (https://doi.org/10.1530/EJE-17-0430)

3 Sachdev V, Matura LA, Sidenko S, Ho VB, Arai AE, Rosing DR \& Bondy CA. Aortic valve disease in Turner syndrome. Journal of the American College of Cardiology 200851 1904-1909. (https://doi. org/10.1016/j.jacc.2008.02.035)

4 Corbitt H, Gutierrez J, Silberbach M \& Maslen CL. The genetic basis of Turner syndrome aortopathy. American Journal of Medical Genetics: Part C, Seminars in Medical Genetics 2019181 117-125. (https://doi. org/10.1002/ajmg.c.31686)

5 Fiot E, Zénaty D, Boizeau P, Haignere J, Dos Santos S, Léger J \& French Turner Syndrome Study Group. X chromosome gene dosage as a determinant of congenital malformations and of age-related comorbidity risk in patients with Turner syndrome, from childhood to early adulthood. European Journal of Endocrinology 2019180 397-406. (https://doi.org/10.1530/EJE-18-0878)

6 Silberbach M, Roos-Hesselink JW, Andersen NH, Braverman AC, Brown N, Collins RT, De Backer J, Eagle KA, Hiratzka LF, Johnson WH et al. Cardiovascular health in Turner syndrome: a scientific statement from the American Heart Association. Circulation: Genomic and Precision Medicine 201811 e000048. (https://doi.org/10.1161/ HCG.0000000000000048)

7 Schoemaker MJ, Swerdlow AJ, Higgins CD, Wright AF, Jacobs PA \& United Kingdom Clinical Cytogenetics Group. Mortality in women with turner syndrome in Great Britain: a national cohort study. Journal of Clinical Endocrinology and Metabolism 200893 4735-4742. (https://doi.org/10.1210/jc.2008-1049)

8 Thunström S, Krantz E, Thunström E, Hanson C, Bryman I \& LandinWilhelmsen K. Incidence of aortic dissection in Turner syndrome. Circulation 2019139 2802-2804. (https://doi.org/10.1161/ CIRCULATIONAHA.119.040552)

9 Carlson M \& Silberbach M. Dissection of the aorta in Turner syndrome: two cases and review of 85 cases in the literature. Journal of Medical Genetics 200744 745-749. (https://doi.org/10.1136/ jmg.2007.052019)

10 Dubois D \& Dubois EF. A formula to estimate the approximate surface area if height and weight be known. 1916. Nutrition 19895 303-311. 
11 Sakamoto I, Sueyoshi E \& Uetani M. MR imaging of the aorta. Magnetic Resonance Imaging Clinics of North America 201018 43-55. (https://doi.org/10.1016/j.mric.2009.09.004)

12 Mortensen KH, Andersen NH \& Gravholt CH. Cardiovascular phenotype in Turner syndrome - integrating cardiology, genetics, and endocrinology. Endocrine Reviews 201233 677-714. (https://doi. org/10.1210/er.2011-1059)

13 Corbitt H, Morris SA, Gravholt CH, Mortensen KH, TippnerHedges R, Silberbach M, Maslen CL \& GenTAC Registry Investigators. TIMP3 and TIMP1 are risk genes for bicuspid aortic valve and aortopathy in Turner syndrome. PLoS Genetics 201814 e1007692 . (https://doi.org/10.1371/journal.pgen.1007692)

14 Mortensen KH, Wen J, Erlandsen M, Trolle C, Ringgaard S, Gutmark EJ, Gutmark-Little I, Andersen NH \& Gravholt CH. Aortic growth rates are not increased in Turner syndrome-a prospective CMR study. European Heart Journal Cardiovascular Imaging 201920 1164-1170. (https://doi.org/10.1093/ehjci/ jez065)

15 Duijnhouwer AL, Bons LR, Timmers HJLM, Van Kimmenade RRL, Snoeren M, Timmermans J, Van Den Hoven AT, Kempers M, Van APJ, Fleischer $\mathrm{K}$ et al. Aortic dilatation and outcome in women with
Turner syndrome. Heart 2019105 693-700. (https://doi.org/10.1136/ heartjnl-2018-313716)

16 Tennyson M. Question 2: in children with Marfan's should we beta block from point of diagnosis? Archives of Disease in Childhood 2018 103 997-1000. . (https://doi.org/10.1136/archdischild-2018-315848)

17 Fuchs MM, Attenhofer Jost C, Babovic-Vuksanovic D, Connolly HM \& Egbe A. Long-term outcomes in patients with Turner syndrome: a 68-year follow-up. Journal of the American Heart Association 20198 e011501. (https://doi.org/10.1161/JAHA.118.011501)

18 Bollache E, Knott KD, Jarvis K, Boubertakh R, Dolan RS, Camaioni C, Collins L, Scully P, Rabin S, Treibel T et al. Two-minute k-space and time-accelerated aortic four-dimensional flow MRI: dual-center study of feasibility and impact on velocity and wall shear stress quantification. Radiology. Cardiothoracic Imaging 20191 e180008. (https://doi.org/10.1148/ryct.2019180008)

19 Prakash SK, Lugo-Ruiz S, Rivera-Dávila M, Rubio N, Shah AN, Knickmeyer RC, Scurlock C, Crenshaw M, Davis SM, Scientific Advisory Board of the TSRR et al. The Turner syndrome research registry: creating equipoise between investigators and participants. American Journal of Medical Genetics, Part C 2019181 135-140. (https://doi.org/10.1002/ajmg.c.31689)

Received 28 March 2020

Revised version received 15 July 2020

Accepted 22 July 2020 